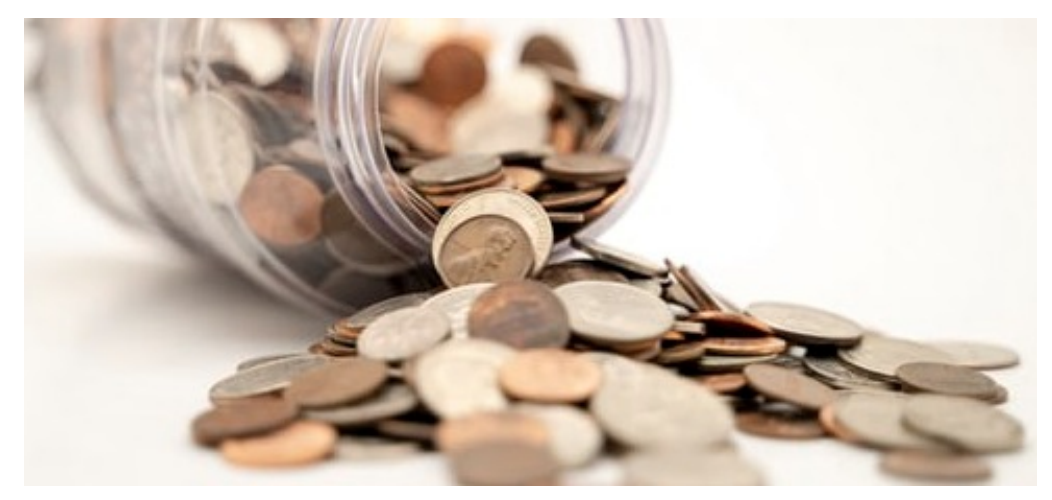

\title{
A Cost Analysis of Overdose Management at a Supervised Consumption Site in Calgary, Canada
}

\author{
Jennifer Jackson \\ 1 University of Calgary
}

Funding: The author(s) received funding from the Faculty of Nursing, University of Calgary.

Potential competing interests: The author(s) declared that no potential competing interests exist.

\section{Abstract}

Background and Aims: We report on a cost study, using population level data to determine the impact of emergency overdose management at supervised consumption services (SCS) versus conventional services.

Design: We completed a cost analysis from a payer's perspective. In this setting, there is a single-payer model of service delivery.

Setting: In Calgary, 'Safeworks Harm Reduction Program,' was established in late 2017 and offers 24/7 access to SCS. The facility is a nurse-led service, available for client drop-in. We conducted a cost analysis for the entire duration of the program. This covers two years and three months.

Measurements: We assessed costs using the following factors, using government health databases: monthly operational costs of providing services for drug consumption, cost of providing EMS for clients with overdoses who could not be revived at the facility, and benefit of EMS costs averted from overdoses that were successfully managed at the SCS.

Findings: The proportion of clients who have overdosed at the SCS has decreased steadily for the duration of the program. The number of overdoses that can be managed on site at the SCS has trended upward, currently $98 \%$. Each overdose that is managed at the SCS produces approximately $\$ 1,600$ CAD in cost savings, with a savings of over $\$ 2.3$ million for the lifetime of the program.

Conclusions: Overdose management at an SCS creates cost savings by offsetting costs required for managing 
overdoses using emergency services.

\section{Introduction}

Supervised consumption services (SCS) are a harm reduction intervention to provide a sanctioned area for clients to use illicit substances, under medical supervision, without crimination (1). A primary aim of SCS is overdose prevention and SCS are widely accepted as decreasing fatal accidental overdoses (2). SCS in Canada began with the creation of Insite in Vancouver in 2003 (1). Before SCS were introduced, conventional services, including ambulance response and emergency departments, are used to support people with overdoses who need medical attention. There is substantial evidence of the benefits of SCS. Insite, in Vancouver, has been widely studied and demonstrated social and economic benefits across a range of measures (1-6). The cost savings relating to HIV infections alone were enough to offset Insite's operating costs $(1,5)$. Estimates related to the prevention of Hepatitis $C$ transmission also showed evidence of significant cost savings (1, 4). Proposals for SCS illustrated projected cost effectiveness in Toronto (7), Victoria (8), Ottawa $(7,9)$, San Francisco (10) and Baltimore (11).

While there are well documented benefits of SCS, studies are limited to a few sites (6). Cost-benefit analyses have tended to focus on HIV prevention or economic benefit of prevented deaths, without including other aspects of the SCS programs (6). There are also challenges in evaluating cost benefits. Clients at SCS may not be required to provide personal health numbers or identifying information, to avoid potential crimination. However, SCS do collect population level data about service use. Our cost analysis study overcomes these limitations by evaluating the population level benefit of decreased use of emergency services, at an SCS site that has not been previously reported in the literature. In this article, we use population level data in a cost analysis, to determine the impact of emergency overdose management at SCS versus conventional services. Please note that all currencies are reported in Canadian dollars. At time of writing, $\$ 1 \mathrm{CAD}=£ 0.59$ GBP.

\section{Methods}

Overdose management was the point of analysis in this study. Overdose (OD) management in this study is defined as the application of a medical intervention to a client who is not rousable, following consumption of an illicit substance. This includes application of oxygen, administration of naloxone, or calling Emergency Medical Services (EMS). Verbally rousing the client, or a client with a 'heavy nod' were excluded from definitions of overdose in this study, limiting the definition of overdose to objectively verifiable information. Oxygen is considered a medical intervention, because the primary cause of overdose related death is hypoxia (12).

\section{Research Site}

In Calgary, a large city in Canada, 'Safeworks Harm Reduction Program,' was established in late 2017 and offers 24/7 access to SCS (13). The facility is a nurse-led service, available for client drop-in. Registered Nurses are always available to reverse an overdose or resuscitate a client. The nurses are equipped to administer oxygen and naloxone, if needed. We conducted a cost analysis for the entire duration of the program. This covers two years and three since the service began. 


\section{Study Design}

This cost analysis was completed from a payer's perspective. A payer in this study refers to the entity (provincial government) providing funding to run the service. The following factors were chosen based on the availability of data to evaluate the cost effectiveness of the site: monthly operational costs of providing service for drug consumption, cost of providing EMS for clients with overdoses who could not be revived at the facility, and benefit of EMS costs averted from overdoses that were successfully managed at the SCS.

\section{Data}

Overdose data was extracted from the monthly, publicly available Government opioid reports (14-16) for the months of November 2017 to January 2020. Some data was imputed, and the methodology is provided in Appendix A.

\section{Analytic methods}

We computed descriptive statistics for the dataset. In addition, we examined the trend in the number of clients who overdosed at the SCS site. To develop an accurate visualization of the data, we have calculated a percentage for the number of overdoses for each month per number of total visits for drug consumption to the SCS, illustrating an overall trend line for overdose rates.

\section{Costs}

The costs were divided into two categories: 1) operating costs for providing services at the SCS and 2) cost of EMS for clients who overdosed at the site but required additional intervention.

\section{Operating Costs at the SCS site}

The first component of the analysis comprised of the operating costs at SCS site. A recent study (13) by the government of Alberta reports that the average cost per visit for drug consumption is $\$ 62.19$. The operating cost at the SCS was calculated by employing the following formula:

$\mathrm{OC}=\mathrm{NDC}(\$ 62.19)$

Where, $\mathrm{OC}$ is the total operating cost and NDC is the number visits to the site for drug consumption each month, from November 2017 to January 2020. This calculation provided the total monthly cost at this site.

\section{Cost of EMS for Overdoses}

The second part of this analysis was the cost of EMS for clients who could not be revived at the SCS site after an overdose. This cost was calculated by employing the following formula:

$\mathrm{EMSC}=\{(\mathrm{TOD})(\$ 385)\}+\{(\mathrm{TOD})(\$ 1,061+\$ 176)\}$ 
Where, EMSC is the total cost of clients needing EMS and TOD is the total number of clients with overdoses taken to EMS for each year. The transport cost of utilizing an ambulance is $\$ 385$ (17) per use.

The cost for an emergency visit for residents of Alberta, Canada is $\$ 1,061$. We used the Comprehensive Ambulatory Classification System (18) grouping methodology resource intensity weight (RIW) and cost of standard hospital stay from the Alberta Health Services to calculate an average cost of an emergency visit due to an overdose. Specific codes for RIW were B184 (Poisoning without Intervention), B204 (Trauma High Resource Intervention), B217 (Trauma with Acute Admission/Transfer without High Resource Intervention), B224(Trauma with Moderate Intervention) and B234 (Poisoning with Minor Intervention).

The cost of physician fees per emergency visit lasting 45 minutes is $\$ 176$ (19).

\section{Benefits}

Calgary's SCS site has not experienced a single overdose related death since its inception, as reported by Alberta Health Services (13). Therefore, we assume that EMS costs are averted due to efficient handling of overdoses at the site.

\section{Benefit from Overdose EMS Averted}

The benefits of managing overdoses at the SCS, without EMS, is calculated by the formula:

$E M S A B=\{(T O D A)(\$ 385)\}+\{(T O D A)(\$ 1,061+\$ 176)\}$

Where, EMSAB is the total benefit from overdose related EMS averted and TODA is the total number of overdoses averted for each year.

\section{Total Benefit, Net Savings, Benefit-Cost Ratios and Cost Effectiveness Ratios}

We added the values for overdose EMS costs that were averted to determine the total benefits of overdose management at the SCS.

\section{Results}

\section{Preliminary Analysis}

Table 1 shows descriptive statistics, including the number of clients, proportion of visits used for drug consumption and referrals for other social services, total number of overdoses, and other related outcomes.

Table 1: Drug Consumption Site Summary Statistics at an SCS site in Calgary, Canada Population Outcomes 
Table 1: Drug Consumption Site Summary Statistics at an SCS site in Calgary, Canada

\begin{tabular}{|c|c|c|c|c|c|c|c|c|}
\hline \multirow{2}{*}{ Population Outcomes } & \multicolumn{2}{|c|}{$2017^{* 1}$} & \multicolumn{2}{|c|}{2018} & \multicolumn{2}{|c|}{2019} & \multicolumn{2}{|c|}{$2020 * 2$} \\
\hline & $\mathrm{n}$ & $\%$ & $\mathrm{n}$ & $\%$ & $\mathrm{n}$ & $\%$ & $\mathrm{n}$ & $\%$ \\
\hline Number of unique clients & \multicolumn{2}{|c|}{540} & \multicolumn{2}{|c|}{7970} & \multicolumn{2}{|c|}{12645} & \multicolumn{2}{|c|}{1270} \\
\hline Total number of visits & 2551 & $100 \%$ & 51791 & $100 \%$ & 69028 & $100 \%$ & 6623 & $100 \%$ \\
\hline Visits used for drug consumption & 2108 & $83 \%$ & 42386 & $82 \%$ & 58629 & $85 \%$ & 5994 & $91 \%$ \\
\hline Visits for referrals to other services & 70 & $3 \%$ & 329 & $1 \%$ & 1969 & $3 \%$ & 153 & $2 \%$ \\
\hline Total overdoses (ODs) & 55 & $100 \%$ & 716 & $100 \%$ & 727 & $100 \%$ & 47 & $100 \%$ \\
\hline ODs provided oxygen & 35 & $64 \%$ & 543 & $76 \%$ & 714 & $98 \%$ & 46 & $98 \%$ \\
\hline ODs provided naloxone & 20 & $36 \%$ & 338 & $47 \%$ & 312 & $43 \%$ & 13 & $28 \%$ \\
\hline OD EMS & 6 & $11 \%$ & 51 & $7 \%$ & 29 & $4 \%$ & 1 & $2 \%$ \\
\hline OD EMS averted & 49 & $89 \%$ & 665 & $93 \%$ & 698 & $96 \%$ & 46 & $98 \%$ \\
\hline
\end{tabular}

$2017^{* 1}$-November and December data

$2020^{* 2}$ - January data

Figure

The proportion of visits for drug consumption has increased over time, but it is notable that nearly $10 \%$ of clients who have accessed the SCS between 2017 and 2020 are not doing so for drug consumption. This finding reinforces the role of the SCS in providing ancillary services, including referrals and wound care. The overall population for drug use at the SCS has increased, while the need for EMS has decreased over time. Presently, 98\% of overdoses are managed on site. This rate has increased steadily since the opening of the SCS. In the most recent full year of operation (2019), 698 overdoses were managed at the SCS, thus avoiding EMS calls.

\section{Overdose trends}

The data shows that the number of overdoses at the site have been increasing over time. However, the number of visits for drug consumption have also increased over time. The linear trend line in Figure 1 shows that the percentage of overdoses declined between November 2017 to January 2020.

Figure 1: Trend analysis of overdoses over the period of Supervised Consumption Site utilization

Figure 1: Trend analysis of overdoses over the period of Supervised Consumption Site utilization

Total Overdose Rates as a Percentage of the Total SCS Population

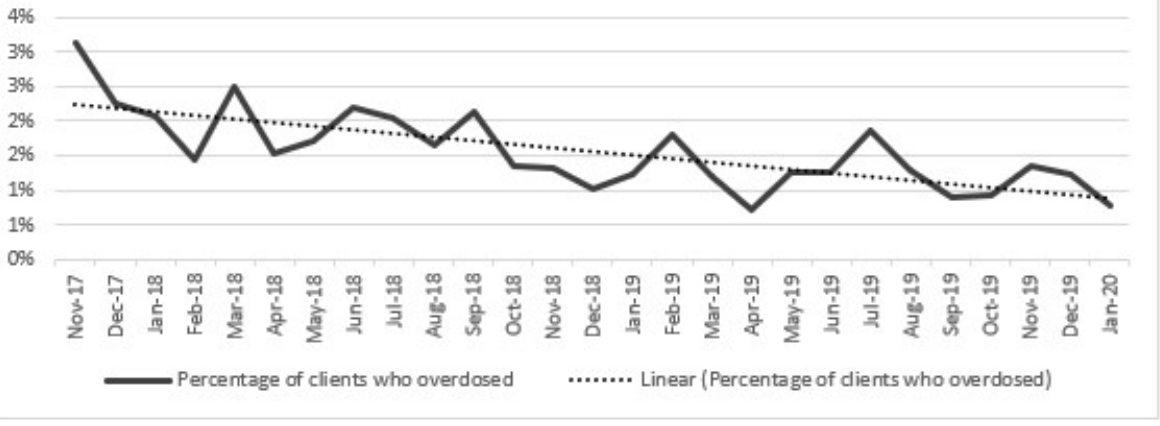

$2017^{* 1}$-Novenmber and December data, 2020*2-January data

Figure 


\section{Costs}

The total costs, which includes the cost of operating the SCS site and the cost of EMS for overdoses, ranged approximately from $\$ 70,000$ to $\$ 374,00$ per month for November 2017 and January 2020 . The annual cost for the most recent full year of operation (2019) was $\$ 3.7$ million. Table 2 provides the total annual and monthly costs. Costs have increased over time, which reflects a corresponding increase in service use.

\section{Benefits}

\section{Benefit from Overdose EMS Averted}

Each overdose that is managed at the SCS produced a benefit of $\$ 1622$ for January 2020 . The benefit of averting the cost of an EMS visit ranges approximately between $\$ 39,000$ and $\$ 94,000$ per month, from November 2017 to January 2020 .

Table 2 provides the total annual and monthly benefits.

Table 2: Total costs and EMS averted Benefits for the SCS site

Table 2: Total costs and EMS averted Benefits for the SCS site

\begin{tabular}{lllllll}
\hline Year & $\begin{array}{c}\text { Operating } \\
\text { Costs }\end{array}$ & $\begin{array}{c}\text { Cost of } \\
\text { OD } \\
\text { EMS }\end{array}$ & $\begin{array}{c}\text { Average } \\
\text { Total } \\
\text { Cost } \\
\text { (Monthly) }\end{array}$ & $\begin{array}{c}\text { Total Cost } \\
\text { (Annual) }\end{array}$ & $\begin{array}{c}\text { Average } \\
\text { EMS } \\
\text { Benefits } \\
\text { (Monthly) }\end{array}$ & $\begin{array}{c}\text { EMS } \\
\text { Benefits } \\
\text { (Annual) }\end{array}$ \\
\hline $\mathbf{2 0 1 7 ^ { * 1 }}$ & $\$ 131,097$ & $\$ 9,732$ & $\$ 70,414$ & $\$ 140,829$ & $\$ 39,739$ & $\$ 79,478$ \\
$\mathbf{2 0 1 8}$ & $\$ 2,635,985$ & $\$ 82,722$ & $\$ 226,559$ & $\$ 2,718,707$ & $\$ 89,886$ & $\$ 1,078,630$ \\
$\mathbf{2 0 1 9}$ & $\$ 3,646,138$ & $\$ 47,038$ & $\$ 307,765$ & $\$ 3,693,176$ & $\$ 94,346$ & $\$ 1,132,156$ \\
$\mathbf{2 0 2 0}^{\star 2}$ & $\$ 372,767$ & $\$ 1,622$ & $\$ 374,389$ & $\$ 374,389$ & $\$ 74,612$ & $\$ 74,612$ \\
\hline
\end{tabular}

$2017^{*_{1}}$-November and December data, 2020*2-January data

Figure

Overall, there were $\$ 2,364,876$ cost savings produced from the overdoses that were managed at the SCS, by avoiding the need for EMS, for the life of the program to date. These costs use the minimum billing fee for the payer and exclude overdose related hospitalization costs, and thus, likely underestimate total costs saved.

\section{Discussion}

This study focuses on a harm reduction service that is part of a complement of programs to address substance misuse, in the context of an opioid crisis. Our study demonstrates that SCS can be justified in part by their benefits in managing overdoses. The declining trend of overdoses indicates the effectiveness of the program in preventing overdoses, as well as managing them. A reduction in the number of overdoses would result in lowering the rate of overdose related deaths and saving more lives, which adds value to the economy and society.

The cost savings of overdose management at the SCS, although substantial, were not sufficient to offset the operating cost of the program. However, this study examined only one aspect of the SCS's potential cost benefits. Several authors found significant cost savings associated with reduced needle sharing at SCS sites $(1,3)$. It is likely that the total cost of this 
SCS could be offset if additional variables were examined. There is potential for further analysis in future studies.

We predict there are secondary benefits, as our study demonstrated that the SCS prevents approximately 700 calls to EMS per year. In addition to the cost savings, it is reasonable to project that other people may be able to access ambulance or emergency department care more quickly, with fewer overdose-related calls for EMS. It has long been known that wait times are a primary determinant of patient satisfaction in emergency departments (20). The SCS in this study prevented notable numbers of patients attending emergency departments each year, which is especially important during COVID-19, where health services are under considerable strain.

Additionally, the SCS site also provides ancillary services, such as referrals for housing. There may be economic and social benefits from clients receiving such additional services, which they may have difficulty accessing elsewhere. Future studies can evaluate the cost benefits of SCS staff supporting clients to access housing and other supportive services. The methodology employed in this study may be applied to evaluate SCS sites at other locations. Overdose management has been considered in prospective cost analyses (7-11) but we have not found other published examples of this analysis with operating SCS.

This study was conducted using available data since the inception of the SCS site in late 2017. Some missing data had to be inferred from similar studies conducted in other provinces and countries. Given the limitations of the anonymous service in this study, more in-depth data on hospitalization and other social service referrals also could not be obtained. Future studies could explore other ways to measure cost-benefit without identifying information from clients.

The study does not employ an economic evaluation methodology due to time and data restrictions. The SCS site is currently under review and have been assigned limited time to present evidence of cost evaluation. As a result, the scope and underlying methodologies are brief and allow limited analysis. Moreover, due to the COVID-19 pandemic, movements are restricted, rendering it infeasible to conduct primary surveys.

This analysis demonstrates the need to conduct economic evaluations of SCS sites. Literature on SCS widely documents the direct benefits of reduced HIV and Hepatitis C infections, skin and soft-tissue infections, economic benefits of overdose related deaths prevented and the indirect benefits of social service referrals; adding these factors to overdose management will improve the analysis.

\section{Conclusion}

In this study, we identified notable cost savings produced through overdose management at SCS. This reduced the reliance on emergency services. In addition, the number of overdoses at the SCS has decreased. The SCS thus offers direct savings, and secondary benefits from fewer visits to emergency departments. Future studies could explore these benefits in more depth. 


\section{References}

1. Bayoumi A. M., Zaric G. S. The cost-effectiveness of Vancouver's supervised injection facility, Canadian Medical Association Journal 2008: 179: 1143-1151.

2. Larson S., Padron N., Mason J., Bogaczyk T. Supervised consumption facilities-Review of the evidence. Retrieved from Philadelphia, PA: Thomas Jefferson University; 2017.

3. Andresen M. A., Boyd N. A cost-benefit and cost-effectiveness analysis of Vancouver's supervised injection facility, International Journal of Drug Policy 2010:21: 70-76.

4. Jozaghi E. A cost-benefit/cost-effectiveness analysis of an unsanctioned supervised smoking facility in the Downtown Eastside of Vancouver, Canada, Harm reduction journal 2014: 11: 30.

5. Pinkerton S. D. Is Vancouver Canada's supervised injection facility cost-saving?, Addiction 2010: 105: $1429-1436$.

6. Caulkins J. P., Pardo B., Kilmer B. Supervised consumption sites: a nuanced assessment of the causal evidence, Addiction 2019: 114: 2109-2115.

7. Enns E. A., Zaric G. S., Strike C. J., Jairam J. A., Kolla G., Bayoumi A. M. Potential cost-effectiveness of supervised injection facilities in Toronto and Ottawa, Canada, Addiction 2016: 111: 475-489.

8. Jozaghi E., Hodgkinson T., Andresen M. A. Is there a role for potential supervised injection facilities in Victoria, British Columbia, Canada?, Urban Geography 2015: 36: 1241-1255.

9. Jozaghi E., Reid A. A., Andresen M. A., Juneau A. A cost-benefit/cost-effectiveness analysis of proposed supervised injection facilities in Ottawa, Canada, Substance Abuse Treatment, Prevention, and Policy 2014: 9: 31-44.

10. Irwin A., Jozaghi E., Bluthenthal R. N., Kral A. H. A cost-benefit analysis of a potential supervised injection facility in San Francisco, California, USA, Journal of Drug Issues 2017: 47: 164-184.

11. Irwin A., Jozaghi E., Weir B. W., Allen S. T., Lindsay A., Sherman S. G. Mitigating the heroin crisis in Baltimore, MD, USA: a cost-benefit analysis of a hypothetical supervised injection facility, Harm Reduction Journal 2017: 14: $29-43$.

12. Marshall B. D., Milloy M. J., Wood E., Montaner J. S., Kerr T. Reduction in overdose mortality after the opening of North America's first medically supervised safer injecting facility: a retrospective population-based study, The Lancet 2011: 377: 1429-1437.

13. Alberta Health. Impact: A socio-economic review of supervised consumption sites in Alberta, Edmonton, AB: Government of Alberta,; 2020, p. 190.

14. Alberta Health. Opioid response surveillance report, Edmonton, Alberta: Government of Alberta; 2019.

15. Alberta Health. Opioid response surveillance report, Edmonton, Alberta: Government of Alberta; 2018.

16. Alberta Health. Opioids and substances of misuse, Edmonton, Alberta: Government of Alberta; 2017.

17. Government of Alberta. Ambulance and emergency health services, Edmonton, Alberta; 2015.

18. Canadian Institute for Health Information. Comprehensive Ambulatory Classification System In: Canadian Institute for Health Information, editor; 2018.

19. McRae A. Alberta billing codes in overdose care, Calgary, Canada: Departments of Emergency Medicine and Community Health Sciences; 2020.

20. Bursch B., Beezy J., Shaw R. Emergency department satisfaction: what matters most?, Annals of Emergency Medicine 1993: 22: 586-591. 
